\title{
An efficient gene deletion procedure for the mushroom-forming basidiomycete Schizophyllum commune
}

\author{
Robin A. Ohm • Jan F. de Jong • Elsa Berends • \\ Fengfeng Wang • Han A. B. Wösten • \\ Luis G. Lugones
}

Received: 25 August 2009/Accepted: 13 February 2010/Published online: 27 February 2010

(C) The Author(s) 2010. This article is published with open access at Springerlink.com

\begin{abstract}
Gene deletion in Schizophyllum commune is hampered by a low incidence of homologous integration. As a consequence, extensive screening is required to identify a transformant with the desired genotype. To alleviate this and to facilitate the assembly of deletion plasmids, vector pDelcas was constructed. This construct has a set of restriction sites, which allows for directional cloning of the flanking sequences at both sides of a nourseothricin resistance cassette. Moreover, it contains a phleomycin resistance cassette elsewhere in the plasmid, which is used to screen for transformants with an ectopic integration of the pDelcas derivative. The use of pDelcas derivatives in combination with an improved PCR screening protocol permitted the efficient identification of $S$. commune deletion strains. This procedure may also function in other basidiomycetes.
\end{abstract}

Keywords Basidiomycete - Schizophyllum commune · Homologous integration · Transformation

Electronic supplementary material The online version of this article (doi:10.1007/s11274-010-0356-0) contains supplementary material, which is available to authorized users.

R. A. Ohm · J. F. de Jong · E. Berends · F. Wang

H. A. B. Wösten · L. G. Lugones ( $\square)$

Microbiology and Kluyver Centre for Genomics of Industrial

Fermentations, Institute of Biomembranes, Utrecht University,

Padualaan 8, 3584, CH, Utrecht, The Netherlands

e-mail: 1.g.lugones@uu.nl

\section{Introduction}

Gene inactivation is an important tool in functional genetics and the most reliable way of implementing it is gene deletion through homologous recombination. The efficiency of this process depends on the pathway that is used by the organism to repair double stranded DNA breaks (Ninomiya et al. 2004). Homologous integration occurs with a high frequency in organisms such as Saccharomyces cerevisae that primarily use the homologous recombination pathway. Plants, animals and filamentous fungi use mainly non homologous end joining for DNA repair. As a result, the most frequent outcome of a DNA transformation is ectopic integration. Ten gene deletions have been reported in the basidiomycete Schizophyllum commune. Gene $X$ of the MATA $\alpha$ mating type locus and the $m t d l$ gene were inactivated with a frequency of $33 \%$ (Marion et al. 1996) and 50\% (Lengeler and Kothe 1999b), respectively. The other genes were deleted with an average frequency of only 3\% (van Wetter et al. 1996, 2000; Robertson et al. 1996; Horton et al. 1999; Lugones et al. 2004; Schubert et al. 2006; Lengeler and Kothe 1999a). The low incidence of homologous integration in most of the cases where a gene was deleted prompted us to set up a screening system for transformants with such an integration event. To this end, pDelcas (accession number GQ184463) was constructed. This vector allows easy directional cloning of flanking sequences at either side of a nourseothricin resistance cassette. The presence of a phleomycin resistance cassette elsewhere in the construct enables elimination of transformants with an ectopic integration due to their resistance to the antibiotic phleomycin. In the second step, phleomycin sensitive colonies are screened using a fast colony PCR protocol to confirm gene inactivation by homologous integration. 


\section{Materials and methods}

\section{Construction of pDelcas}

Vector pDelcas consists of a backbone of pUC20 encompassing the $S$. commune phleomycin and nourseothricine resistance cassettes (Schuren and Wessels 1994; van Peer et al. 2009) (Fig. 1). Flanking regions of 1.5-2 kb of the gene of interest can be cloned at either side of the nourseothricin resistance cassette by replacing a $2.4 \mathrm{~kb}$ Van91I fragment and a $0.64 \mathrm{~kb}$ SfiI fragment, respectively. Van91I and SfiI recognize the sequences CCANNNNNTGG and GGCCNNNNNGGCC, respectively, and both cut within the variable middle part. This allows the generation of tailored sticky ends. As a result, fragments cut with SfiI (which is chosen because it is an 8 cutter whose recognition sites rarely occur in genomic DNA) can be directionally cloned into a vector cut with either Van91I or SfiI. To this end, both genomic flanking sequences of the gene of interest are amplified with primers that contain a SfiI site. The forward and reverse primers should have SfiI sites that differ in their variable region, resulting in different sticky

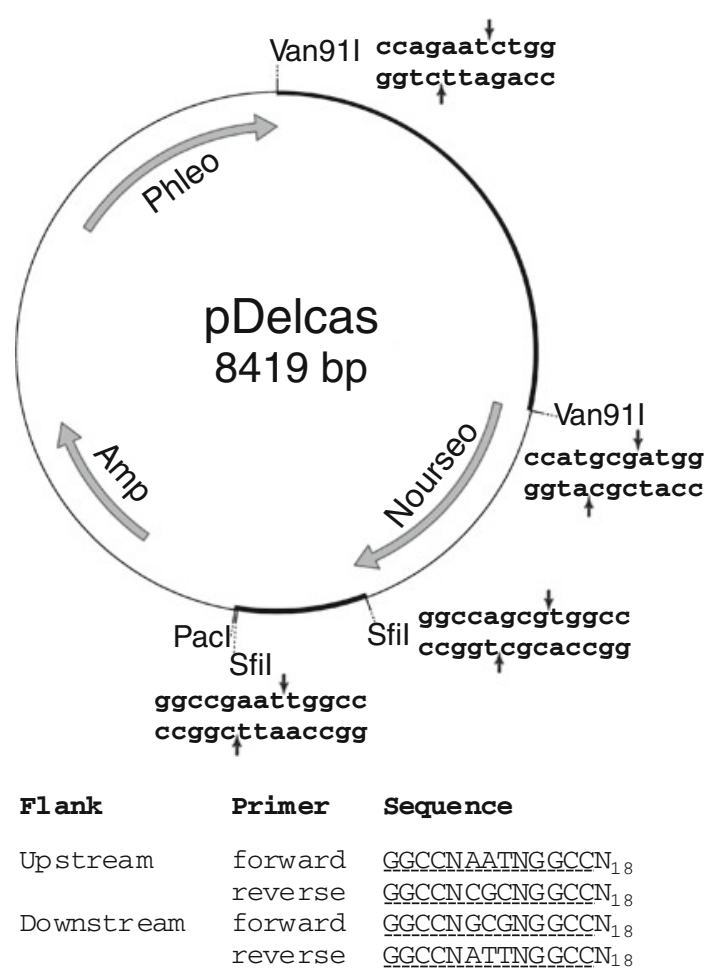

Fig. 1 Vector pDelcas is based on a pUC20 backbone. It contains a phleomycin and a nourseothricin resistance cassette. Van91I and SfiI sites flank fragments from bacteriophage $\lambda$ of 2,4 and $0.64 \mathrm{~kb}$, respectively (indicated in bold). Custom made sticky ends in these sites allow directional cloning when combining the primers depicted in the lower panel. The underlined sequence of the primers represent the recognition site of SfiI, while the $\mathrm{N}_{18}$ region represent the flank specific sequence ends (Fig. 1). In the first step, one of the flanks with the SfiI sticky ends is directionally cloned in the Van91I sites of pDelcas. In the second step, the other flank is cloned in the SfiI sites. In the case a restriction site for SfiI is present within one of the flanking sequences, primers can be used that contain sites for BstAPI (GCANNNNNTGC), DraIII (CACNNNTGC) or Van91I (CCANNNNNTGG) and that obey the rules for the variable region as depicted in Fig. 1.

Transformation and first screening

Circular DNA of derivatives of pDelcas was introduced in $S$. commune strain 4-8 (MATA43 MATB41, Fowler et al. 1999) according to van Peer et al. (2009). Selection was done on minimal medium (Dons et al. 1979) with $8 \mu \mathrm{g} \mathrm{ml}^{-1}$ nourseothricin (Jena Biosciences, Jena, Germany). Transformants were transferred to a second selection plate with nourseothricin and then screened on minimal medium plates with $25 \mu \mathrm{g} \mathrm{ml}^{-1}$ phleomycin (Cayla S.A.R.L., Toulouse, France). Transformants that failed to grow on phleomycin were candidates to have a gene deletion due to homologous integration resulting from a double cross-over.

\section{PCR screening}

From the transformants that failed to grow on phleomycin, a small piece of mycelium $\left(2-25 \mathrm{~mm}^{2}\right)$ was taken from a colony that had grown on a nourseothricin selection plate. The fragment was placed in a $2 \mathrm{ml}$ Eppendorf tube together with a new metal ball (Marabu, 3/16" or approximately $5 \mathrm{~mm}$ in diameter). The mycelium was frozen in liquid nitrogen and homogenized for 1 min at $25 \mathrm{~Hz}$ using a Retsch Tissue Lyser II. One $\mathrm{ml}$ of CTAB-buffer (2\% CTAB, 0.1 M Tris- $\mathrm{HCl}$ pH 8.0, 1.4 M NaCl) was added to the powered mycelium, after which the metal ball was removed with a magnet. The tubes were placed at $65^{\circ} \mathrm{C}$ for $20 \mathrm{~min}$. Cell debris was pelleted by centrifugation for $1 \mathrm{~min}$ at $20,000 \mathrm{~g}$ and half volume chloroform was added to the supernatant. After mixing, the samples were centrifuged for $5 \mathrm{~min}$ at $20,000 \mathrm{~g} .800 \mu \mathrm{l}$ of the water (upper) phase was transferred to a clean tube and DNA was precipitated by adding $640 \mu \mathrm{l}$ isopropanol. After centrifugation for $5 \mathrm{~min}$ at $20,000 \mathrm{~g}$, the pellet was washed with $500 \mu \mathrm{l} 70 \%$ ethanol and dissolved in $50 \mu \mathrm{l}$ TE buffer. For PCR, $1 \mu \mathrm{l}$ of the DNA was mixed with primers, Taq polymerase, Taq buffer (as supplied by the manufacturer), $1.5 \%$ DMSO, $1.5 \mathrm{mM} \mathrm{MgCl} 2$ and $200 \mu \mathrm{M}$ of each nucleotide. For screening, primers were used that only render a product in case of homologous integration of the flanking sequences of the deletion construct (Fig. 2). The forward primer pr1 anneals to the genomic DNA immediately outside the upstream flank. Its sequence depends on the gene that is inactivated. The reverse primer $\operatorname{pr}^{\prime}{ }^{\prime}$ anneals in 

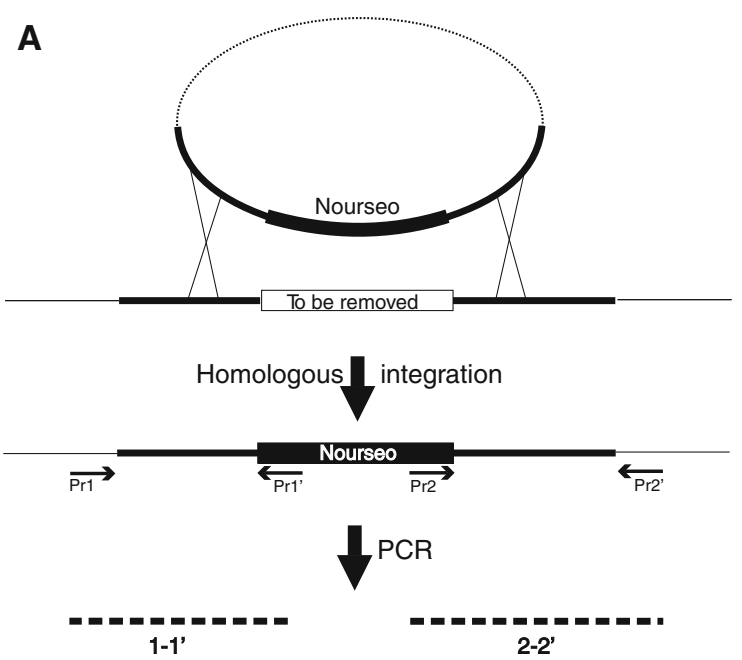

Fig. 2 Screening of transformants in which a pDelcas derivative has been introduced. Integration will be either homologous (a) or ectopic (b). PCR with three primer pairs reveals which integration event has

the nourseothricin resistance cassette and is used for screening of all gene deletions. In the same way primers pr2 (anneals in the nourseothricin resistance cassette) and pr2' (gene specific) are used to check the downstream flank. As a negative control, a PCR is performed with primers pr1 and $\mathrm{pr} 3^{\prime}$ (anneals in the deleted fragment of the gene). Generally, 35 PCR cycles were sufficient to amplify a specific band. Agar from the medium did not inhibit the PCR.

\section{Crossings}

Derivatives of strain 4-8 with a gene inactivation were crossed with the compatible isogenic strain 4-8b (MATA41 MATB43). Segregation of nourseothricin resistance in the F1 was assessed to exclude multiple integrations (integration in a single locus should give a 1:1 ratio of resistant and sensitive colonies). Single integrations were confirmed by Southern analysis. Crossing was also used to clean heterokaryons that harbor transformed and untransformed nuclei. Such heterokaryons can result from fusion of transformed and non-transformed (regenerating) protoplasts and give PCR products in both the positive and negative controls.

\section{Results and discussion}

A pDelcas derivative was constructed with the flanking regions of a 524 bp coding region fragment of myn6 (Protein ID: 114035; http://genome.jgi-psf.org/Schco1/Schco1.

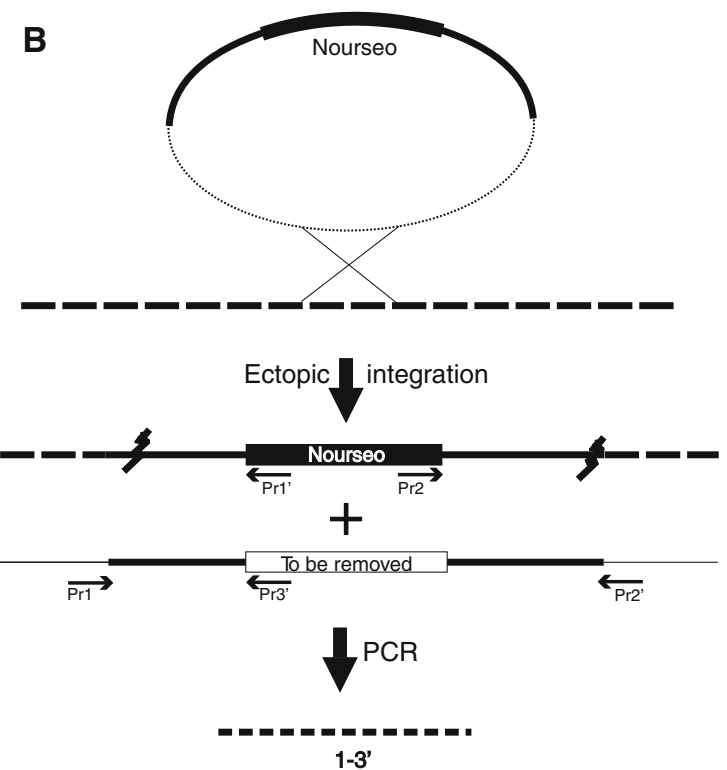

occurred. A third possibility not depicted here is homologous integration which resulted from a single crossing over. In this case product $1-1^{\prime}$ or $2-2^{\prime}$ will be found together with product $1-3^{\prime}$

home.html) that encodes a putative transcription factor. Primers MYN6_ufw, MYN6_urv, MYN6_dfw and MYN6_ drv (Table 1) were used to amplify up and downstream flanks, respectively. These flanking regions were cloned into pDelcas resulting in vector pDelcas-MYN6updw. 309 nourseothricin resistant colonies were obtained after introduction of the construct in S. commune. Of these, 22 were sensitive to phleomycin. Phleomycin sensitivity may be due to a homologous integration of the flanking regions due to a double cross-over. Alternatively, it may be caused by an ectopic integration which took place within the phleomycin resistance cassette. To discriminate between these events, PCR was performed using three primer pairs (Fig. 2; Table 1). Genomic DNA of one of the transformants gave a band of $1,338 \mathrm{bp}$ with primers SC3TerminatorPr and MYN6_outrev and a band of $1,805 \mathrm{bp}$ with primers

Table 1 Primers used in this study to delete myn6

\begin{tabular}{ll}
\hline Primer & Sequence $\left(5^{\prime}-3^{\prime}\right)$ \\
\hline MYN6_ufw & GGCCGAATCGGCCCGCCTTCTCCAG \\
MYN6_urv & GGCCACGCTGGCCCAAGACACCTC \\
MYN6_dfw & GGCCAGCGGGGCCTTCGCACGTC \\
MYN6_drv & GGCCGATTAGGCCGTCCTTTGCTTTC \\
MYN6_outfw (pr1) & CCGCCAGGGTACAACTACTC \\
Nourdelrev (pr1') & TTGGTGACCTCCAGCCAGAG \\
SC3TerminatorPr & GGCTGAGTCGTGGACTAAAG \\
(pr2) & \\
MYN6_outrev (pr2') & GGGCGGACGAGATGTACAAG \\
MYN6_control (pr3) & TATAGCTCTCGAGCGGCGTCAGAGATG
\end{tabular}


Table 2 Results of the screening of transformants from four different gene deletion experiments

\begin{tabular}{lcclll}
\hline Gene & $\begin{array}{l}\text { Nour(+) } \\
\text { colonies }\end{array}$ & $\begin{array}{l}\text { Nour(+) } \\
\text { Phleo(-) colonies }\end{array}$ & $\begin{array}{l}\text { Nour(+) Phleo(-) colonies } \\
(\% \text { of Nour(+) colonies) }\end{array}$ & $\begin{array}{l}\text { Transformants } \\
\text { with a gene } \\
\text { deletion }\end{array}$ & $\begin{array}{l}\text { Transformants } \\
\text { with a gene deletion } \\
(\% \text { of Nour(+) Phleo(-) colonies) }\end{array}$ \\
\hline myn6 & 309 & 22 & 7.1 & 1 & 4.5 \\
spc33 & 495 & 140 & 28.3 & 2 & 1.4 \\
reg1 & 68 & 16 & 23.5 & 1 & 6.3 \\
sc15 & 76 & 20 & 26.3 & 1 & 5.0 \\
Average percentage & & & 21.3 & & 4.3 \\
\hline
\end{tabular}

MYN6_outfw and nourdelrev. These bands are explained by the homologous recombination between pDelcasMYN6updw and the myn6 locus. Primers MYN6_outfw and MYN6_control did not result in a 1,150 bp fragment, which is expected when the myn6 fragment has been deleted. Southern analysis of chromosomal DNA confirmed the deletion of myn6 (Supplementary Figure 1). A fragment of 1,913 bp hybridized with the wild-type DNA, whereas a $3,428 \mathrm{bp}$ fragment hybridized in the transformant. Like gene myn6, three other genes have been inactivated using pDelcas (Table 2). In general, 20-25\% of the nourseotricin resistant colonies are phleomycin sensitive. Of these transformants, about $4 \%$ have a gene deletion. Recently, we found that up to $60 \%$ of the false positives can be eliminated by decreasing the concentration of phleomycin during the screening to $5 \mu \mathrm{g} \mathrm{ml}^{-1}$. By using this concentration also transformants are eliminated that express the phleomycin resistance cassette to a low extent. The remaining false positives can be explained by a single integration event within the phleomycin resistant cassette.

The use of simultaneous selection for transformation and homologous integration would simplify the screening even more. Kothe et al. (1993) suggested the use of trpl (involved in tryptophan synthesis) as a marker for ectopic integration. In their proposed setup the deletion construct is transformed to a $\mathrm{trpl}^{-} \mathrm{frll}^{-}$strain that shows fluorescence under UV exposure as a consequence of anthranilic acid accumulation. Colonies with an ectopic integration are not fluorescent because the product of trpl processes anthranilic acid to tryptophan. Unfortunately, this strategy implies the use of a strain with two mutations. In contrast, pDelcas can be used in any strain.

Recently, we described that the number of S. commune transformants can be increased tenfold by adding a low non-selective concentration of phleomycin in the regeneration medium (van Peer et al. 2009). This increase can be explained by the fact that this antibiotic introduces double strand breaks in the DNA. We have also observed that the low concentration of phleomycin promotes single integrations. However, addition of a low concentration of phleomycin in the regeneration medium did not improve efficiency of gene deletion using pDelcas. In fact, no gene deletions were observed in nearly a thousand transformants that had been transformed with pDelcas derivatives. These data and the fact that phleomycin increases expression of genes involved in non homologous end joining (van Peer et al. 2009) indicates that this antibiotic promotes ectopic integrations.

Efficient gene deletion protocols are essential for functional gene analysis. The genomes of the mushroom forming fungi Phanerochaete chrysosporium, Coprinopsis cinerea, Laccaria bicolor and S. commune have been sequenced and those of Pleurotus ostreatus and Agaricus bisporus will be ready soon. Vector pDelcas and the described PCR procedure allow rapid screening of transformants in $S$. commune. Possibly, the same procedure can also be used for deletion of genes in other basidiomycetes. This is indicated by the fact that expression cassettes of $S$. commune are functional in other basidiomycetes like $P y c$ noporus cinnabarinus (Alves et al. 2004), Agaricus bisporus and Pleurotus ostreatus (L.G. Lugones, unpublished).

Acknowledgments This research is supported by the Dutch Technology Foundation STW, applied science division of NWO and the Technology Program of the Ministry of Economic Affairs.

Open Access This article is distributed under the terms of the Creative Commons Attribution Noncommercial License which permits any noncommercial use, distribution, and reproduction in any medium, provided the original author(s) and source are credited.

\section{References}

Alves AMCR, Record E, Lomascolo A, Scholtmeijer K, Asther M, Wessels JGH, Wösten HAB (2004) Highly efficient production of laccase by the basidiomycete Pycnoporus cinnabarinus. Appl Environ Microbiol 70:6379-6384

Dons JJM, de Vries OMH, Wessels JGH (1979) Characterization of the genome of the basidiomycete Schizophyllum commune. Biochim Biophys Acta 563:100-112

Fowler TJ, DeSimone SM, Mitton MF, Kurjan J, Raper CA (1999) Multiple sex pheromones and receptors of a mushroom-producing fungus elicit mating in yeast. Mol Biol Cell 10:2559-2572

Horton JS, Palmer GE, Smith WJ (1999) Regulation of dikaryonexpressed genes by FRT1 in the basidiomycete Schizophyllum commune. Fungal Genet Biol 26:33-47 
Kothe E, Kothe HW, Specht CA, Novotny CP, Ullrich RC (1993) The frl gene, a useful system for rapid screening of tryptophan auxotrophs in Schizophyllum commune. Mycologia 85: 381-384

Lengeler KB, Kothe E (1999a) Identification and characterization of brt1, a gene down-regulated during B-regulated development in Schizophyllum commune. Curr Genet 35:551-556

Lengeler KB, Kothe E (1999b) Mated: a putative peptide transporter of Schizophyllum commune expressed in dikaryons. Curr Genet 36:159-164

Lugones LG, de Jong JF, de Vries OMH, Jalving R, Dijksterhuis J, Wösten HAB (2004) The SC15 protein of Schizophyllum commune mediates formation of aerial hyphae and attachment in the absence of the SC3 hydrophobin. Mol Microbiol 53: 707-716

Marion AL, Bartholomew KA, Wu J, Yang H, Novotny CP, Ullrich RC (1996) The A $\alpha$ mating-type locus of Schizophyllum commune: Structure and function of gene $X$. Curr Genet 29:143-149

Ninomiya Y, Suzuki K, Ishii C, Inoue H (2004) Highly efficient gene replacements in Neurospora strains deficient for non-homologous end-joining. Proc Natl Acad Sci USA 101:12248-12253
Robertson CI, Bartholomew KA, Novotny CP, Ulrich RC (1996) Deletion of the Schizophyllum commune A $\alpha$ locus: the roles of $\mathrm{A} \alpha Y$ and $Z$ mating-type genes. Genetics 144:1437-1444

Schubert D, Raudaskoski M, Knabe N, Kothe E (2006) Ras GTPaseactivating protein Gap1 of the homobasidiomycete Schizophyllum commune regulates hyphal growth orientation and sexual development. Eukaryot Cell 5:683-695

Schuren FHJ, Wessels JGH (1994) Highly-efficient transformation of the homobasidiomycete Schizophyllum commune to phleomycin resistance. Curr Genet 26:179-183

van Peer AF, de Bekker C, Vinck A, Wösten HAB, Lugones LG (2009) Phleomycin increases transformation efficiency and promotes single integrations in Schizophyllum commune. Appl Environ Microbiol 75:1243-1247

van Wetter MA, Schuren FHJ, Schuurs TA, Wessels JGH (1996) Targeted mutation of the SC3 hydrophobin gene of Schizophyllum commune affects formation of aerial hyphae. FEMS Microbiol Lett 140:265-269

van Wetter MA, Wösten HAB, Wessels JGH (2000) SC3 and SC4 hydrophobins have distinct roles in formation of aerial structures in dikaryons of Schizophyllum commune. Mol Microbiol 36: 201-210 